\title{
Critical appraisal of the role of pegloticase in the management of gout
}

This article was published in the following Dove Press journal:

Open Access Rheumatology: Research and Reviews

26 June 2012

Number of times this article has been viewed

\author{
Hang-Korng Ea ${ }^{1,2}$ \\ Pascal Richette ${ }^{1,2}$ \\ 'Hôpital Lariboisière, Rheumatology \\ Department, Paris, France; ${ }^{2}$ University \\ of Paris Diderot, Sorbonne Paris Cité, \\ Paris, France
}

\begin{abstract}
Gout is a debilitating disease secondary to chronic hyperuricemia, and the subsequent deposition of monosodium urate crystals is responsible for acute flare, gout arthropathies, tophi and renal lithiasis. Uric acid is the end product of purine metabolism in humans because the gene encoding uricase was lost during hominoid evolution. Pegloticase is a recombinant mammalian uricase conjugated to polyethylene glycol that catalyzes the oxidation of uric acid into allantoin, a more soluble end product. The use of this drug as urate-lowering therapy is a new approach in treating severe gout refractory to conventional therapy with xanthine oxidase inhibitors and uricosuric agents. Intravenous pegloticase has potent and long-lasting urate-lowering capacity with rapid efficacy on tophi resolution. However, pegloticase treatment is associated with infusion-related reactions despite prevention therapy with high-dose corticosteroids. Exacerbation of pre-existing cardiovascular diseases is another concern. The mechanisms of these events are unknown. Caution with long-term use of pegloticase is warranted, especially for patients with cardiovascular diseases.
\end{abstract}

Keywords: gout, urate-lowering therapy, pegloticase, uricase, urate oxidase, immunogenicity

\section{Introduction}

Gout is secondary to monosodium urate (MSU) crystal deposition occurring after longterm hyperuricemia defined as a serum urate (SU) level $>6.8 \mathrm{mg} / \mathrm{dL}(415 \mu \mathrm{mol} / \mathrm{L})$, which corresponds to the physiologic saturation threshold of urate. Uric acid (UA) is the end product of purine metabolism in humans because of the inactivation of the gene that encodes for uricase during hominoid evolution in the Miocene. ${ }^{1}$ Thus, the SU level is close to its saturation threshold in human blood and is 50 times higher than that in other mammals. High SU level had some advantages for human evolution, but can also lead to pathological conditions such as gout and cardiovascular diseases. Gout is a disabling condition, and MSU crystal deposition can result in recurrent acute flare, gout arthropathies, tophi, and kidney stones. Hyperuricemia and gout are frequently associated with several medical comorbidities, including metabolic syndrome, which encompasses hypertension, diabetes mellitus, hyperlipidemia and obesity, as well as chronic renal failure and cardiovascular diseases. According to European League Against Rheumatism (EULAR) recommendations, gout management includes treating acute flare, lowering SU concentration to $<6 \mathrm{mg} / \mathrm{dL}(360 \mu \mathrm{mol} / \mathrm{L})$, and treating medical comorbidities. ${ }^{2}$

Urate-lowering therapy (ULT) is indicated in all patients with recurrent flare, gout arthropathies, tophi, and kidney stones. Until recently, ULT was based on inhibiting xanthine 
oxidase only (with allopurinol and febuxostat) and uricosuric agents (probenecid, benzbromarone, and sulfinpyrazone). Allopurinol and febuxostat are the sole approved and available ULT agents in Europe, and treatment with allopurinol, the most widely prescribed, often fails to achieve the target of $<6 \mathrm{mg} / \mathrm{dL}$ (360 $\mu \mathrm{mol} / \mathrm{L}$ ) because of renal failure that limits the dosage. However, Stamp et al have recently highlighted that carefully increasing the dose of allopurinol is effective in lowering $\mathrm{SU}<6 \mathrm{mg} / \mathrm{dL}$ in up to $89 \%$ of patients, and even in patients with renal impairment. ${ }^{3}$ Moreover, these authors suggest that monitoring serum oxypurinol level, the active metabolite of allopurinol, should be useful to, first, identify noncompliant patients and, second, adapt allopurinol dose for patient needs. ${ }^{4,5}$ Thus, allopurinol failure appears to be more secondary to inappropriate dose and nonadherence than ineffectiveness. ${ }^{4,6}$ Nevertheless, uricase agents represent an interesting alternative in ULT $\operatorname{arsenal}^{7}$ (Figure 1). In 2010, the US Food and Drug Administration (FDA) approved pegloticase (KRYSTEXXA ${ }^{\circledR}$; Savient Pharmaceuticals, East Brunswick, NJ) a PEGylated uric acid-specific enzyme, for treating chronic gout in adults with disease refractory to conventional therapy. ${ }^{8}$

\section{Uricase as potent ULT}

Urate oxidase (also known as uricase) converts urate to allantoin, which is substantially more soluble than urate and readily excreted. Although urate oxidase was thought the only enzyme involved in this reaction, conversion of UA to allantoin was recently found to occur by sequential chemical modifications catalyzed by urate oxidase, 5-hydroxyisourate (5-HIU) hydrolase, and 2-oxo-4-hydroxy-4-carboxy-5ureidoimidazoline (OHCU) decarboxylase. ${ }^{9,10}$ Thus, UA is oxidized to 5-HIU, which is hydrolyzed to OHCU, which is decarboxylated to S-(+)-allantoin (Figure 1). In humans, the 5-HIU hydrolase gene has several inactivating mutations, and the OHCU decarboxylase gene has not been found in transcriptome data. ${ }^{9} 5$-HIU is an unstable product that spontaneously hydrolyzes into OHCU, which is also spontaneously decarboxylated into racemic allantoin. However, urate oxidation produces racemic allantoin over a period of hours, whereas the complete enzymatic components produce dextrorotatory allantoin in seconds. Thus, treatment with uricase alone can result in transient accumulation of 5-HIU. In addition, UA oxidation is associated with the formation of $\mathrm{H}_{2} \mathrm{O}_{2}$, a reactive oxygen species.

Initial studies of rasburicase (Elitek/Fasturtec ${ }^{\circledR}$; sanofiaventis, Bridgewater, NJ), a recombinant form of urate oxidase from Aspergillus flavus, developed for treating tumor lysis syndrome in children with cancer, indicated the potential of such enzymes to lower serum urate levels in patients with gout. ${ }^{11}$ However, the use of rasburicase for gout is

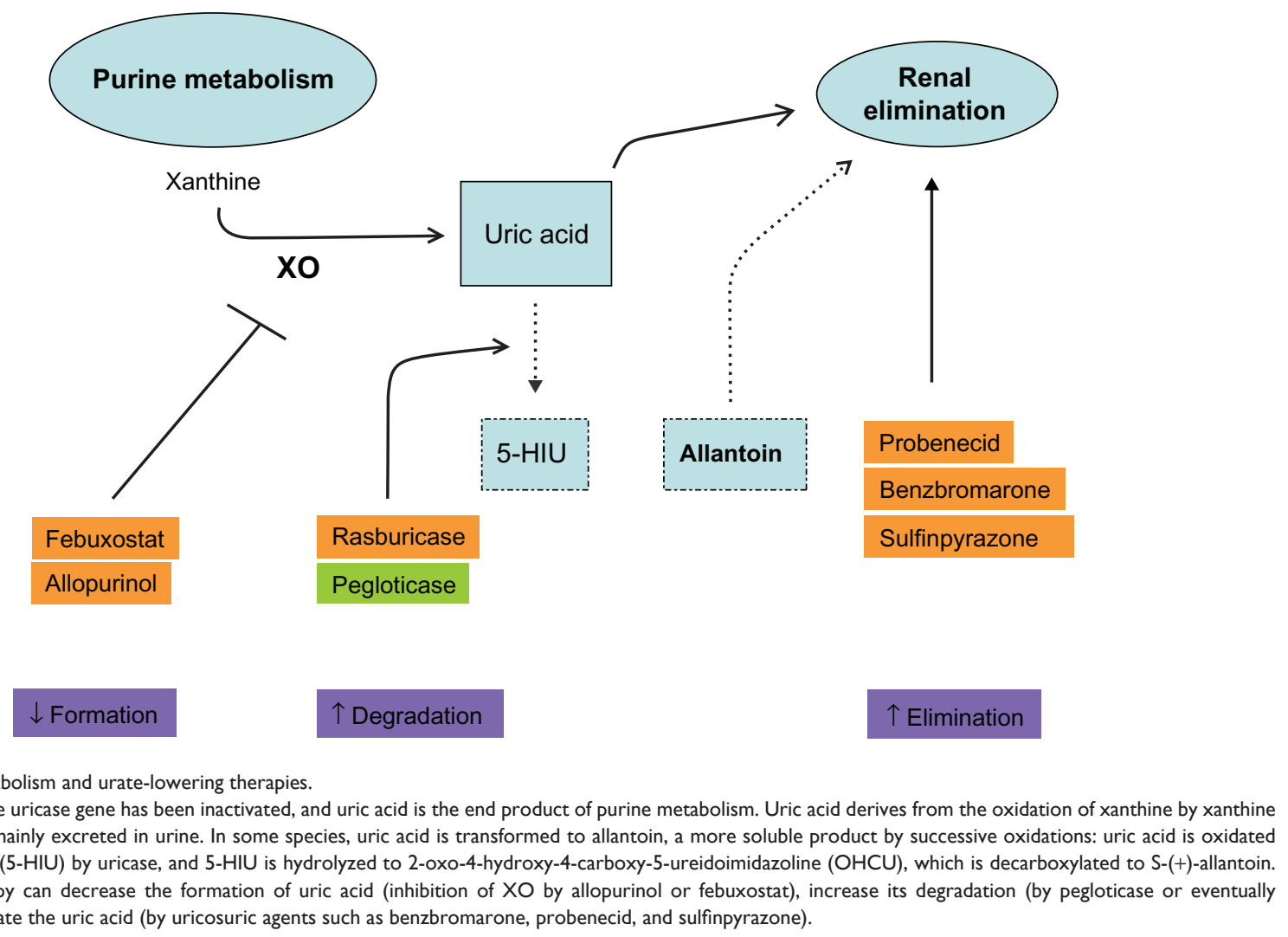


limited by its immunogenicity and relatively short half-life. ${ }^{11}$ To overcome these problems, a PEGylated recombinant mammalian uricase, pegloticase, was developed.

\section{Pegloticase (KRYSTEXXA ${ }^{\circledR}$ ) Pharmacodynamics and pharmacokinetics}

Pegloticase is a recombinant mammalian uricase (porcine/ baboon variant) produced in Escherichia coli and a tetrameric enzyme. Each subunit is conjugated with several strands of a 10-kDa monomethoxypoly(ethylene glycol) (mPEG). The rationale for the addition of $\mathrm{mPEG}$ to this molecule was to reduce the potential for immunogenicity and increase circulation half-life compared to the non-PEGylated porcine enzyme. ${ }^{8}$ Pegloticase has no known secondary pharmacodynamic activities besides its capacity for oxidating UA. ${ }^{8}$

No pharmacodynamic drug interaction studies have investigated pegloticase. However, because pegloticase features the formation of anti-PEG antibodies that bind to the PEG portion, it may have potential for binding with other PEGylated products. The impact of anti-PEG antibodies on patient responses to other PEG-containing therapeutics is unknown. ${ }^{8}$ Much of the immunogenicity of PEG-protein conjugates with $\mathrm{mPEG}$ is due to the methoxy groups. ${ }^{12}$

In a Phase II multidose open-label study, intravenous pegloticase, $8 \mathrm{mg}$ every 2 weeks for 12 to 14 weeks, was the most effective in lowering $\mathrm{SU}$, with the highest proportion of responders $(87.5 \%)$ and the highest percentage of time without hyperuricemia (91.9\%). ${ }^{13}$ Subsequent double-blind, placebo-controlled, randomized clinical trials (RCTs) confirmed the efficacy of pegloticase, $8 \mathrm{mg}$ for 2 weeks, in terms of ULT and rapid resolution of tophi. ${ }^{14}$

In a Phase I study of a single perfusion of pegloticase of 4 to $8 \mathrm{mg}$, the mean SU level decreased from $11.1 \pm 0.6 \mathrm{mg} / \mathrm{dL}$ at baseline to $<2 \mathrm{mg} / \mathrm{dL}$ within 24 hours and to a nadir of $1.0 \pm 0.5 \mathrm{mg} / \mathrm{dL}$ within 48 to 72 hours postinfusion. ${ }^{15}$ Moreover, 6 hours after pegloticase perfusion, SU level was $<6 \mathrm{mg} / \mathrm{dL}$. ${ }^{13}$ Thus, the onset of pegloticase activity is rapid, and in the Phase III RCT, the mean SU levels were $0.7 \mathrm{mg} / \mathrm{dL} 24$ hours after the first infusion of $8 \mathrm{mg}$ compared with $8.2 \mathrm{mg} / \mathrm{dL}$ in the placebo group. ${ }^{14}$ Maximum serum concentrations of pegloticase (based on measurements of plasma uricase activity) were dose-proportional after a single infusion of pegloticase. ${ }^{15}$ The uricase activity of pegloticase is maintained for several weeks after one infusion. Thus, with doses every 2 weeks, the mean serum concentrations of pegloticase were higher after dose 6 than after dose 1 , which suggests some accumulation of pegloticase. The mean half-life of uricase activity was $9.2 \pm 3.2$ days (range
6.4-13.8 days). ${ }^{13,15}$ The intravenous pegloticase half-life was about 14 days (range 7-44 days)..$^{16}$ The half-life of rasburicase is 15.5 to 22.5 hours. ${ }^{17}$

In a Phase II open-label study of pharmacodynamic and pharmacokinetic properties of pegloticase in 41 patients with refractory gout, mean half-maximal inhibitory concentration and $90 \%$ inhibitory concentration values were 0.10 and $0.93 \mu \mathrm{g} / \mathrm{mL}$, respectively. Thus, low levels of pegloticase are sufficient to provide $50 \%$ and $90 \%$ of the maximal suppressive effect of pegloticase. ${ }^{16}$ These values are easily achieved by the currently approved regimen. Indeed, across all regimens in the Phase II multidose open-label study, the median maximum concentration for pegloticase was 0.80 to $5.96 \mu \mathrm{g} / \mathrm{mL}$ after the first or last infusion, at 4 or $8 \mathrm{mg}$, with treatment every 2 weeks, or 8 or $12 \mathrm{mg}$ every 4 weeks, and was reached within 24 hours after administration. ${ }^{17}$ Similarly, the authors observed pegloticase values that ranged from undetectable to $8.887 \mu \mathrm{g} / \mathrm{mL}$ with different dosing regimens, including 4 and $8 \mathrm{mg}$ every 2 weeks or 8 and $12 \mathrm{mg}$ every 4 weeks. ${ }^{16}$ Monte Carlo simulations revealed that $8 \mathrm{mg}$ every 2 weeks or every 4 weeks was effective in maintaining SU levels $<6 \mathrm{mg} / \mathrm{dL}$ for 18 months. ${ }^{16}$ Monte Carlo simulations involved data for 160 patients receiving four different regimens of pegloticase: $8 \mathrm{mg}$ every 2 weeks for 18 months or every 2 weeks for 6 months, then every 4 weeks for 12 months; or 8 mg every 4 weeks for 18 months or every 4 weeks for 6 months, then every 2 weeks for 12 months. ${ }^{16}$

The clearance and volume of distribution of pegloticase (about 5-10 L) are influenced by body surface area and antipegloticase antibodies, but its pharmacokinetic profile is not affected by age, sex, body weight, or creatinine clearance. ${ }^{15}$ The effects of renal or hepatic impairment on the pharmacokinetics of pegloticase have not been formally studied. ${ }^{8}$

\section{Efficacy and safety}

Clinical data on the safety and efficacy of pegloticase for treating severe gout in patients with disease refractory to conventional therapy are from eight clinical studies: two Phase I studies, ${ }^{15,18}$ two Phase II studies, ${ }^{13,16}$ two replicate pivotal Phase III placebo-controlled trials, ${ }^{14}$ and a completed Phase IIIb open-label extension (OLE) study. ${ }^{19}$ A small, open-label Phase II study evaluating oxidative status secondary to prolonged and deep UA decrease with pegloticase clearly demonstrated the rapid and spectacular effect of pegloticase as ULT. ${ }^{20}$ Except for the first Phase I study, which evaluated safety and efficacy after subcutaneous injection of pegloticase, all other clinical studies investigated safety and efficacy after intravenous administration. 
The two 6-month, randomized, double-blind, placebocontrolled, multicenter, North American (United States, Canada, and Mexico) Phase III trials, involving 212 adult patients with chronic gout refractory to conventional therapy ${ }^{14}$ gave the most conclusive data. Inclusion criteria were baseline SU at least $8.0 \mathrm{mg} / \mathrm{dL}(480 \mu \mathrm{mol} / \mathrm{L})$, symptomatic gout with at least three self-reported gout flares during the previous 18 months or at least one tophus or gouty arthropathy and a history of allopurinol contraindication or failure to normalize SU despite at least 3 months' treatment with the maximal medically appropriate dose of allopurinol. Unfortunately, the dose of allopurinol was not available in this article. A total of 212 participants were randomized to receive pegloticase ( $8 \mathrm{mg}$ by intravenous infusion every 2 or 4 weeks) or placebo in a 2:2:1 ratio. Baseline characteristics were similar across the trials and treatment groups: the mean duration of gout was 12 to 16 years, mean SU levels were 9.4 to $10.4 \mathrm{mg} / \mathrm{dL}$, tophi were present in $65 \%$ to $79 \%$ of patients, metabolic and renal disorders were common, and cardiovascular comorbidities or risk factors were present in more than $80 \%$ of patients.

Pegloticase was found to be a rapid and potent ULT, decreasing SU to $<6 \mathrm{mg} / \mathrm{dL}$ within 24 hours after the first infusion in all treated patients. The proportion of patients achieving the primary endpoint (SU level $<6 \mathrm{mg} / \mathrm{dL}$ for more than $80 \%$ of the time during months 3 and 6 ) was $42 \%$ and $35 \%$ with $8 \mathrm{mg}$ pegloticase every 2 and 4 weeks, respectively, as compared with $0 \%$ in the placebo group $(P<0.001)$. Moreover, the efficacy of pegloticase was associated with a greater proportion of complete tophi resolution (40\% and $21 \%$ with $8 \mathrm{mg}$ every 2 and 4 weeks, respectively) compared with placebo (7\%); significant clinical benefit was seen in tender joint and swollen joint count and patient global assessment. ${ }^{14}$ The dosage of $8 \mathrm{mg}$ every 2 weeks rather than $8 \mathrm{mg}$ every 4 weeks gave a higher proportion of sustained responders (defined as SU level $<6 \mathrm{mg}$ / $\mathrm{dL}$ from starting treatment throughout the trials; $61.2 \% \mathrm{vs}$ $51.2 \%$ ). This effect is probably related to the formation of anti-pegloticase antibodies and the infusion-related reactions (IRRs, see below). Finally, all studies showed that a single pegloticase perfusion could maintain the SU level $<6 \mathrm{mg} / \mathrm{dL}$ between 14 and more than 35 days according to "patient responder status." ${ }^{13-15,20}$ For $76.2 \%$ (16/21) of persistent responders, the mean SU level was $5.7 \pm 3.0 \mathrm{mg} / \mathrm{dL} 5$ weeks after the last infusion, whereas for transient responders, the mean SU returned to baseline level $(10.8 \pm 1.3 \mathrm{mg} / \mathrm{dL})$ by the second to fourth perfusion. ${ }^{20}$ Similarly, in the RCT reported by Sundy and colleagues, for $43.8 \%$ (74/169) of transient responders, urate-lowering efficacy was lost by week 5 with monthly treatment and by weeks 7 to 10 with biweekly treatment. ${ }^{14}$ In fact, for persistent responders, SU levels were $<2 \mathrm{mg} / \mathrm{dL}$ with biweekly treatment after the first infusion and throughout the first 6 months. For persistent responders with monthly treatment, SU levels were between 2 and $4 \mathrm{mg} / \mathrm{dL}$ during the study. ${ }^{14}$ In contrast, for transient responders, SU levels were $>4 \mathrm{mg} / \mathrm{dL}$ as soon as week 3. The same results were observed by Hershfield and colleagues: for persistent responders receiving $8 \mathrm{mg}$ every 3 weeks, the mean SU level was $0.9 \pm 0.5 \mathrm{mg} / \mathrm{dL}$ throughout the 18 weeks of the study. ${ }^{20} \mathrm{~A}$ post-hoc analysis showed loss of urate-lowering efficacy was significantly associated with high antipegloticase antibody titers. ${ }^{14}$ Hershfield and colleagues observed the same phenomena ${ }^{20}$ (see Tolerance and safety). The long-term efficacy of pegloticase was suggested by the OLE study: 151/157 (96\%) of enrolled patients chose to receive pegloticase ( $8 \mathrm{mg}$ every 2 weeks $[n=82]$ or 4 weeks $[n=67])$ or observation only $(n=2)$. The choice was made blindly. Data for 121 and 95 patients are available for more than 12 and 18 months of treatment, respectively. In addition, data for up to 2.6 years for some endpoints are available for a subgroup of 79 patients. ${ }^{17,19,21}$ In all, 50 of 60 (83\%) persistent responders in the Phase III RCT showed a sustained SU level of $<6 \mathrm{mg} / \mathrm{dL}$ during the 30-month study. ${ }^{17,21}$ As well, the proportion of patients with tophus resolution increased during the studies: 79 patients had 308 tophi in the RCT trial, and 29 (34\%) experienced total resolution during the study; 24 of 79 (30\%) patients in the OLE trial experienced target tophus resolution. ${ }^{21}$ Finally, other chronic gout-related outcomes were sustained or further ameliorated, including reduction from baseline in number of tender joints and improvement in Health Assessment Questionnaire disability index and pain score, as well as SF-36 physical component summary score..$^{17,19,21}$

\section{Tolerance and safety Gout flare}

Gout flare was the most common adverse event for $75 \%$ to $81 \%$ of patients receiving pegloticase. ${ }^{14}$ Gout flare was more frequent with pegloticase than placebo ( $78 \%$ vs $53 \%$ ) during the first 3 months of treatment and was less frequent (48.5\% vs $67 \%$ ) between 3 and 6 months. Gout flare is usual (if not necessary) and a well-known phenomenon occurring at the onset of every ULT; it is due to rapid lowering of serum urate levels and subsequent mobilization/dissolution of MSU crystals accumulated in tissue. Gout flare prophylaxis relies on low-dose colchicine or nonsteroidal anti-inflammatory 
drug intake during the first 6 months of ULT or until the disappearance of tophi. ${ }^{2,22,23}$

\section{Infusion-related reactions}

The major side effect with pegloticase is IRR, occurring in $26 \%$ to $53.8 \%$ of cases depending on the regimen and in $26 \%$ to $37.5 \%$ for the currently approved dosage ( $8 \mathrm{mg}$ every 2 weeks). ${ }^{13,14}$ IRR represents the most common reason for discontinuation of pegloticase $(10 \%$ for biweekly treatment and $13 \%$ for monthly in the phase III RCT), reaching $29.2 \%$ across all regimens in the multidose Phase II RCT. ${ }^{13,14}$ IRRs most commonly manifest as urticaria $(10.6 \%)$, chest discomfort $(9.5 \%)$, chest pain $(9.5 \%)$, erythema $(9.5 \%)$, pruritus (9.5\%), and dyspnea (7.1\%); it occurs at any time during treatment and mostly occurs (91\%) during infusion. ${ }^{8}$ IRRs are thought to result from the release of various cytokines. For instance, in the Phase III RCT, serum tryptase levels were increased in 12 of 108 IRRs $(10.2 \%) .{ }^{14}$ Slowing the rate of infusion or stopping the infusion and restarting it at a slower rate reduced the manifestation of some IRRs. IRRs were considered serious for 5\% (biweekly treatment) and $8 \%$ (monthly) of patients, ${ }^{14}$ and anaphylaxis was retrospectively identified in 14 of 273 patients (5.1\%) in the clinical trial. ${ }^{8}$ The frequency of anaphylaxis was $6.5 \%$ and $4.8 \%$ for the fortnightly regimen (8/123 patients) and monthly regimen (6/126), respectively. ${ }^{8}$ There were no cases of anaphylaxis among patients receiving placebo. Anaphylaxis may occur in any infusion, including a first infusion, and generally manifests within 2 hours of the infusion. However, delayedtype hypersensitivity reactions have been reported. Such reactions occurred in patients receiving pre-treatment with an oral antihistamine, intravenous corticosteroid (200 mg hydrocortisone), or acetaminophen. Thus, corticosteroids must be maintained before each pegloticase infusion, but can result in specific complications and be associated with some serious adverse events. ${ }^{24}$ The US FDA clinical criteria for diagnosing anaphylaxis were skin or mucosal tissue involvement, plus airway compromise and/or reduced blood pressure with or without associated symptoms, and a temporal relationship with pegloticase or placebo infusion with no other identifiable cause. ${ }^{8,25}$

Importantly, the risk of IRRs was higher for patients who had the lost urate-lowering effect (ie, those with SU level $>6 \mathrm{mg} / \mathrm{dL}) .{ }^{8,14}$ Indeed, a post hoc analysis of the Phase III RCT results indicated that patients with an IRR had SU levels $>6.0 \mathrm{mg} / \mathrm{dL}$ before the first IRR (20/22 [91\%] and 24/34 [71\%] receiving pegloticase, $8 \mathrm{mg}$ every 2 and 4 weeks, respectively). Thus, SU levels should be monitored before each infusion, and treatment discontinuation should be considered with $\mathrm{SU}>6 \mathrm{mg} / \mathrm{dL} .^{8,14}$ Similarly, the presence of antipegloticase antibodies was associated with IRRs. ${ }^{14}$ In the Phase III RCT, antipegloticase antibodies were detected in 134 of 150 evaluable patients (89\%) receiving pegloticase. The incidence of IRRs was significantly greater for 52 patients with high antipegloticase antibody titers $(>1 / 2430)$ at any time during the study than for 84 patients who never had high antipegloticase antibody titers $(60 \%$ vs $19 \% ; P<0.001){ }^{14}$

\section{Antipegloticase antibodies}

The development of antipegloticase antibodies occurs frequently in patients receiving pegloticase, reaching $89 \%$ in the Phase III RCT, between $63 \%$ and $86 \%$ in the Phase II multidose RCT, and $41 \%$ in the intravenous Phase I study. ${ }^{13-15}$ Interestingly, antibodies were directed more often against the mPEG component rather than the recombinant uricase. ${ }^{15}$ Pegloticase antibody was of immunoglobulin $\mathrm{M}$ ( $\mathrm{IgM})$ and IgG isotypes (usually the IgG2 subclass, but also IgG3) and did not neutralize pegloticase activity in vitro except for one patient. ${ }^{13-15}$ The presence of anti-PEG antibodies is associated with decreased pegloticase half-life compared with no anti-PEG antibodies (11.0 [range 4-21] vs 16.1 [range 4-22] days), a lower proportion of responders than nonresponders and a lower proportion of time with $\mathrm{SU}<6 \mathrm{mg} / \mathrm{dL}$ during the treatment period. ${ }^{13,15,20}$ Moreover, as stated above, high titers of antipegloticase antibodies $(>1 / 2430)$ are associated with IRRs and loss of urate-lowering efficacy. Thus, urate-lowering efficacy was maintained in only $2 \%(1 / 52)$ of patients with a high level of antipegloticase antibodies as compared with $63 \%$ (52/82) who never had high antibody titers. ${ }^{14}$ The effect of anti-PEG antibodies on other pegylatedtherapeutic agents is unknown.

\section{Cardiovascular adverse events}

Cardiovascular adverse events (AEs) are another concern during pegloticase treatment. Deaths and AEs that may be due to cardiovascular factors were assessed with the Antiplatelet Trialists' Collaboration (APTC) composite of endpoints. ${ }^{14,26}$ The number of patients with cardiovascular AEs receiving pegloticase was fourfold greater, although not significantly, than that for the placebo group..$^{14}$ During the Phase III RCT study and follow-up, there were seven deaths (four with pegloticase treatment and three with placebo). The four deaths with pegloticase involved cardiac arrest, cardiac arrhythmia, renal failure, and sepsis. In addition to these fatal cases, one patient receiving pegloticase experienced myocardial infarction. 
All three cases with APTC cardiovascular AEs had four or more cardiovascular risk factors at baseline. ${ }^{14}$ In all, two $(2.3 \%)$, six $(7.1 \%)$, and zero patients who received $8 \mathrm{mg}$ pegloticase every 2 weeks, $8 \mathrm{mg}$ every 4 weeks, and placebo, respectively, experienced serious non-APTC cardiovascular AEs (including chronic heart failure, arrhythmia, deep vein thrombosis, transient ischemic attack, unstable angina, and coronary revascularization). ${ }^{14}$ Mechanisms of these cardiovascular AEs were not clear, and the role of high-dose corticosteroids before each pegloticase infusion could not be ruled out. ${ }^{24}$ In addition, all of these events occurred in patients with preexisting comorbid risk factors for cardiovascular events. Thus, the occurrence of the AEs was not unexpected, given the high prevalence of underlying cardiovascular disease in the patients who participated in these trials. Therefore, pegloticase should not be given to patients with cardiac dysfunction such as arrhythmia, left ventricular dysfunction, cardiac failure, or coronary artery disease and should be limited in duration to avoid AEs. The studies excluded patients with unstable coronary artery disease, arrhythmia, uncompensated congestive heart failure, and uncontrolled hypertension. ${ }^{13-15,20}$

\section{Miscellaneous AEs}

Other AEs that occurred with $\geq 5 \%$ frequency were nausea (10\%), vomiting (5\%), constipation (6\%), nasopharyngitis $(7 \%)$, contusion or ecchymosis $(11 \%)$, chest pain $(6 \%)$, and dyspnea (6\%). ${ }^{8,14}$ The multidose and Phase III OLE studies evidenced nephrolithiasis (15\%), peripheral edema (14.0\%), arthralgia (12\%-17\%), anemia (10\%), headache (10\%), muscle spasms $(10 \%)$, pyrexia $(10 \%)$, back pain $(7 \%-10.5 \%)$, diarrhea (7\%-12.5\%), upper respiratory tract infection (10.5\%), sinusitis $(10.5 \%)$, cough $(10.5 \%)$, urinary tract infection (8.8\%), fatigue (7\%), and pruritus $(7 \%){ }^{13,17,19}$

\section{Unresolved questions in pegloticase therapy}

Because of the recent development of pegloticase as a ULT, several questions remain unresolved. What is the real impact of persistent low SU level (concentration equal to species with effective uricase), because the loss of the uricase gene has had some advantages in human evolution? Specifically, how is oxidative status (dys)regulated, as well as blood pressure? What is the effect on aging and cancer development? What is(are) the effect(s) of $\mathrm{H}_{2} \mathrm{O}_{2}$ generated by UA oxidation? Similarly, what is(are) the effect(s) of transient accumulation of 5-HIU in humans? Indeed, in mice, with active uricase, loss-of-function mutation of
5-HIU hydrolase leading to 5-HIU accumulation induced hepatocellular carcinoma. ${ }^{27}$ UA metabolites may be toxic, and urate oxidase activity without 5-HIU hydrolase function may affect liver growth and transformation. The absence of 5-HIU hydrolase in humans predicts slowed metabolism of 5-HIU after treatment with exogenous urate oxidase. Thus, prolonged uricase therapy should be combined with careful assessment of toxic effects associated with extrahepatic production of uric acid metabolites. ${ }^{27}$

\section{Place of pegloticase in ULT}

In the United States, intravenous pegloticase, $8 \mathrm{mg}$ every 2 weeks, is indicated for chronic gout in adults with disease refractory to conventional therapy (ie, chronic gout in patients with abnormal SU level and with signs and symptoms inadequately controlled by xanthine oxidase inhibitors at the maximal medically appropriate dosage or for whom these drugs are contraindicated). This drug is currently not available in Europe. With allopurinol, the first-line choice for ULT in patients with gout according to NICE, ${ }^{11,23}$ the target SU level is often not reached, and the drug might be associated with serious allergic side effects. However, recent data suggest that even in patients with impaired renal function, in up to $89 \%$ of patients, target levels of SU can be reached with careful uptitration of the allopurinol dose. ${ }^{3}$ Moreover, these authors show that monitoring oxypurinol serum levels is helpful in allopurinol therapy. ${ }^{5}$ Thus, there are correlations between allopurinol dose, plasma oxypurinol, and SU levels. The relationship between allopurinol dose and oxypurinol plasma level is affected by renal impairment. A plasma oxypurinol concentration of $100-150 \mu \mathrm{mol} / \mathrm{L}$ is required to achieve a target $\mathrm{SU}$ level $<6 \mathrm{mg} / \mathrm{dL}(360 \mu \mathrm{mol} / \mathrm{L})$. In patients who fail to attain adequate urate lowering with allopurinol, measurement of plasma oxypurinol will aid in assessing compliance with the dosing regimen. Furthermore, carefully increasing the allopurinol dose until plasma oxypurinol is $>100 \mu \mathrm{mol} / \mathrm{L}$ may achieve the SU level target. ${ }^{5}$ Altogether, these results suggest that monitoring oxypurinol is useful in assessing allopurinol efficacy and adherence. Further studies are warranted in order to confirm these results and, subsequently, the first place of allopurinol in ULT.

Patients with gout, for whom allopurinol is contraindicated or is ineffective, now receive febuxostat. A recent European review suggested that EULAR target levels of SU can be achieved in $66 \%$ to $88 \%$ of patients receiving the highest approved daily dose of $120 \mathrm{mg}$ febuxostat. ${ }^{28}$ In the US, the maximal approved dose of febuxostat is $80 \mathrm{mg} /$ day and has been shown to have similar efficacy as $120 \mathrm{mg} /$ day. ${ }^{29}$ 
Benzbromarone is a powerful uricosuric that at 100 to $200 \mathrm{mg}$ /day can be effective in achieving EULAR target SU levels in $80 \%$ to $100 \%$ of patients, respectively, ${ }^{30,31}$ but its use is restricted in many European countries because of the possible risk of rare but serious hepatotoxicity. ${ }^{32}$ Other uricosuric drugs such as probenecid and sulfinpyrazone are less effective, especially in patients with mild renal impairment.

Gouty patients with disease refractory or intolerant to xanthine oxidase inhibitors (allopurinol and febuxostat) and uricosuric agents are candidates for treatment with intravenous pegloticase. Whether pegloticase could be used as first-line ULT for patients with severe gout (with tophi, arthropathies, or UA lithiasis) is unknown. Here, pegloticase could be started first for a short period of time (ie, 6 months or until the disappearance of tophi), and then followed by a xanthine oxidase inhibitor or novel uricosuric. This scheme needs further investigation. However, until the availability of these data, allopurinol should still be considered as a first-line ULT.

\section{Disclosures}

HKE reports no conflicts of interest. PR has received fees from Savient, Ipsen, Menarini, Novartis, and Sobi.

\section{References}

1. Wu XW, Lee CC, Muzny DM, Caskey CT. Urate oxidase: primary structure and evolutionary implications. Proc Natl Acad Sci U S A. 1989;86:9412-9416.

2. Zhang W, Doherty M, Bardin T, et al. EULAR evidence based recommendations for gout. Part II: Management. Report of a task force of the EULAR Standing Committee for International Clinical Studies Including Therapeutics (ESCISIT). Ann Rheum Dis. 2006;65:1312-1324.

3. Stamp LK, O'Donnell JL, Zhang M, et al. Using allopurinol above the dose based on creatinine clearance is effective and safe in patients with chronic gout, including those with renal impairment. Arthritis Rheum. 2011;63:412-421.

4. Keith MP, Gilliland WR. Improving the use of allopurinol in chronic gout: monitoring oxypurinol levels to guide therapy. Clin Pharmacol Ther. 2011;90:363-364.

5. Stamp LK, Barclay ML, O’Donnell JL, et al. Relationship between serum urate and plasma oxypurinol in the management of gout: determination of minimum plasma oxypurinol concentration to achieve a target serum urate level. Clin Pharmacol Ther. 2011;90:392-398.

6. Sarawate CA, Brewer KK, Yang W, et al. Gout medication treatment patterns and adherence to standards of care from a managed care perspective. Mayo Clin Proc. 2006;81:925-934.

7. Garay RP, El-Gewely MR, Labaune JP, Richette P. Therapeutic perspectives on uricases for gout. Joint Bone Spine. 2012;79(3): 237-242.

8. US Food and Drug Administration. Pegloticase: full prescribing information. 2010. Available from: http://dailymed.nlm.nih.gov/dailymed/ drugInfo.cfm?id=41481. Accessed October 17, 2011.

9. Ramazzina I, Folli C, Secchi A, Berni R, Percudani R. Completing the uric acid degradation pathway through phylogenetic comparison of whole genomes. Nat Chem Biol. 2006;2:144-148.

10. Tipton PA. Urate to allantoin, specifically (S)-allantoin. Nat Chem Biol. 2006;2:124-125.
11. Richette P, Briere C, Hoenen-Clavert V, Loeuille D, Bardin T. Rasburicase for tophaceous gout not treatable with allopurinol: an exploratory study. J Rheumatol. 2007;34:2093-2098.

12. Sherman MR, Williams LD, Sobczyk MA, Michaels SJ, Saifer MG. Role of the methoxy group in immune responses to $\mathrm{mPEG}$-protein conjugates. Bioconjug Chem. 2012;23:485-499.

13. Sundy JS, Becker MA, Baraf HS, et al. Reduction of plasma urate levels following treatment with multiple doses of pegloticase (polyethylene glycol-conjugated uricase) in patients with treatment-failure gout: results of a phase II randomized study. Arthritis Rheum. 2008;58: 2882-2891.

14. Sundy JS, Baraf HSB, Yood RA, et al. Efficacy and tolerability of pegloticase for the treatment of chronic gout in patients refractory to conventional treatment: two randomized controlled trials. JAMA. 2011;306:711-720.

15. Sundy JS, Ganson NJ, Kelly SJ, et al. Pharmacokinetics and pharmacodynamics of intravenous PEGylated recombinant mammalian urate oxidase in patients with refractory gout. Arthritis Rheum. 2007;56:1021-1028.

16. Yue CS, Huang W, Alton M, et al. Population pharmacokinetic and pharmacodynamic analysis of pegloticase in subjects with hyperuricemia and treatment-failure gout. J Clin Pharmacol. 2008;48:708-718.

17. Lyseng-Williamson KA. Pegloticase: in treatment-refractory chronic gout. Drugs. 2011;71:2179-2192.

18. Ganson NJ, Kelly SJ, Scarlett E, Sundy JS, Hershfield MS. Control of hyperuricemia in subjects with refractory gout, and induction of antibody against poly(ethylene glycol) (PEG), in a phase I trial of subcutaneous PEGylated urate oxidase. Arthritis Res Ther. 2006;8:R12.

19. Hamburger SA, Lipsky PE, Khanna D, Simon L. Safety and efficacy of long-term pegloticase (KRYSTEXXA_) Pegloticase treatment in adult patients with chronic gout refractory to conventional therapy [abstract no OP0113]. 12th Annual Congress of the European League Against Rheumatism; May 25-28, 2011; London.

20. Hershfield MS, Roberts LJ 2nd, Ganson NJ, et al. Treating gout with pegloticase, a PEGylated urate oxidase, provides insight into the importance of uric acid as an antioxidant in vivo. Proc Natl Acad Sci US A. 2010;107:14351-14356.

21. Baraf HS, Gutierrez-Urena S, Vazquez-Mellado J, et al. Progressive reduction in tophus burden with pegloticase therapy in patients with chronic gout refractory to conventional therapy [abstract]. Arthritis Rheum. 2010;62 Suppl 10:157. DOI: 10.1002/art.27926.

22. Richette P, Bardin T. Gout. Lancet. 2010;375:318-328.

23. Terkeltaub RA. Clinical practice. Gout. $N$ Engl J Med. 2003;349: 1647-1655.

24. Ea HK, Chales G, Liote F. Pegloticase and chronic gout. JAMA. 2011;306:1979; author reply, 1979-1980.

25. Sampson HA, Munoz-Furlong A, Campbell RL, et al. Second symposium on the definition and management of anaphylaxis: summary report - Second National Institute of Allergy and Infectious Disease/ Food Allergy and Anaphylaxis Network symposium. J Allergy Clin Immunol. 2006;117:391-397.

26. Collins R, Baigent C, Sandercock P, Peto R. Antiplatelet therapy for thromboprophylaxis: the need for careful consideration of the evidence from randomised trials. Antiplatelet Trialists' Collaboration. BMJ. 1994;309:1215-1217.

27. Stevenson WS, Hyland CD, Zhang JG, et al. Deficiency of 5-hydroxyisourate hydrolase causes hepatomegaly and hepatocellular carcinoma in mice. Proc Natl Acad Sci U S A. 2010;107:16625-16630.

28. Jansen TL, Richette P, Perez-Ruiz F, et al. International position paper on febuxostat. Clin Rheumatol. 2010;29:835-840.

29. Becker MA, Schumacher HR Jr, Wortmann RL, et al. Febuxostat compared with allopurinol in patients with hyperuricemia and gout. N Engl J Med. 2005;353:2450-2461.

30. Reinders MK, Haagsma C, Jansen TL, et al. A randomised controlled trial on the efficacy and tolerability with dose escalation of allopurinol $300-600 \mathrm{mg}$ /day versus benzbromarone $100-200 \mathrm{mg} /$ day in patients with gout. Ann Rheum Dis. 2009;68:892-897. 
31. Reinders MK, van Roon EN, Jansen TL, et al. Efficacy and tolerability of urate-lowering drugs in gout: a randomised controlled trial of benzbromarone versus probenecid after failure of allopurinol. Ann Rheum Dis. 2009;68:51-56.
32. Jansen TL, Reinders MK, van Roon EN, Brouwers JR. Benzbromarone withdrawn from the European market: another case of "absence of evidence is evidence of absence"? Clin Exp Rheumatol. 2004; $22: 651$.

\section{Publish your work in this journal}

Open Access Rheumatology Research and Reviews is an international, peer-reviewed, open access journal, publishing all aspects of clinical and experimental rheumatology in the clinic and laboratory including the following topics: Pathology, pathophysiology of rheumatological diseases; Investigation, treatment and management of rheumatological diseases; Clinical trials and novel pharmacological approaches for the treatment of rheumatological disorders. The manuscript management system is completely online and includes a very quick and fair peerreview system, which is all easy to use. Visit http://www.dovepress.com/ testimonials.php to read real quotes from published authors. 